\title{
Comparação de Métodos Alternativos para Estimar a Produção de Leite de Vacas da Raça Gir ${ }^{1}$
}

\section{Tarcísio de Moraes Gonçalves ${ }^{2}$, Mário Luiz Martinez ${ }^{3}$, Cláudio Manoel Rodrigues de Melo, Rui da Silva Verneque ${ }^{3}$, Antonio Ilson Gomes de Oliveira ${ }^{5}$}

RESUMO - O objetivo deste trabalho foi comparar métodos alternativos para estimar a produção de leite de vacas da raça Gir. Um total de 3632 lactações de vacas da raça Gir, no período de 1986 a 1996, foi analisado. Os métodos foram: A - controle na ordenha da manhã realizado nos meses ímpares e o da ordenha da tarde nos meses pares - a produção da ordenha da manhã foi multiplicada pelo fator multiplicativo da manhã (FM), e a da tarde pelo fator multiplicativo da tarde (FT), B e D - controle realizado na ordenha da manhã (em B a produção da manhã foi multiplicada pelo FM e em D produção foi duplicada); C e E - controle realizado na ordenha da tarde (em C produção da tarde foi multiplicada pelo FT e em E foi duplicada). Todos os métodos foram comparados com a produção real (R). O método A mostrou-se mais preciso, sendo a precisão do método C intermediária entre os métodos B e D. Correlações de Pearson entre as produções estimadas pelos diferentes métodos e R, pela produção até 305 dias e pela produção total foram, respectivamente, 0,98 ; 0,95; e 0,96 para A, 0,97; 0,93; e 0,95 para B, 0,93; 0,90; e 0,89 para C, 0,97; 0,94; e 0,95 para D e 0,93; 0,89; e 0,89 para E. O método A mostrou-se o mais viável para estimar a produção de leite no dia do controle leiteiro, por sua praticidade e precisão, enquanto os metodos $\mathrm{C}$ e $\mathrm{E}$ foram os piores entre os metodos de controle avaliados.

Palavras-chave: bovinos, gado de leite, métodos de controle, produção de leite, raça Gir

\section{Comparison of Alternative Methods to Estimate the Milk Yield of Cows from Gyr Breed}

ABSTRACT - The objective of this work was to compare the alternative methods to estimate the milk yield of cows from Gyr breed. A total of 3,632 lactations from of Gyr breed cows, from 1986 to 1996, were analyzed. The methods were: A - the morning milking control was performed in the odd months and the afternoon milking control in the even months - the yield of the morning milking control was multiplied by the multiplicative factor of the morning (MF) and that of evening by the multiplicative factor of the afternoon (AF), B and D - the control was performed in the morning milking (in B, the yield of the morning milking was multiplied by the MF and in D the yield was multiplied by two); $\mathrm{C}$ and $\mathrm{E}$ - the control was performed in the evening milking (the yield of the afternoon milking was multiplied by the AF and in E it was multiplied by two). All methods were compared with the actual production (R). The method A showed the most precise, being the precision of method $\mathrm{C}$ intermediate between the B and D methods. The Pearson correlation among the estimate yields by the different methods and R, by the production at the 305 days and total production, were, respectively, .98,.95, and .96 for $\mathrm{A}, .97, .93$, and .95 for $\mathrm{B}, .93, .90$, and .89 for $\mathrm{C}, .97, .94$, and .95 for D and $.93, .89, .98$ for E method. The method A showed the most viable to estimate the milk production in the day of milk production control by its practicability and precision, while the $\mathrm{C}$ and E methods were the worst among the evaluated milk control methods.

Key Words: cattle, dairy cattle, control method, milk yield, Gyr breed

\section{Introdução}

São poucas as propriedades onde se faz o controle da produção de leite. Alguns criadores costumam medir, com pouca freqüência, a produção, a qual informa apenas quanto o animal está produzindo naquele momento. Outros controlam somente as melhores produtoras, o que pode ser útil para fins de propaganda, mas não é adequado para seleção, pois requer o controle de todo rebanho. Quando as vacas são alimentadas em função da produção individual, os erros na estimação da produção no dia do controle são importantes.

Entretanto, na avaliação de reprodutores, erros nos controles individuais, que podem ser compensados no cálculo da produção total por lactação, são

\footnotetext{
${ }^{1}$ Parte da Monografia apresentada pelo terceiro autor à Universidade Federal de Lavras (UFLA), como parte das exigências do Programa Especial de Treinamento (PET/CAPES).

2 Professor do Departamento de Zootecnia da Universidade Federal de Lavras (UFLA). DZO da UFLA, Campus da UFLA, Cx. Postal 37, 37200 - 000, Lavras - MG, e.mail - tarcísio@ufla.br

3 Pesquisador da EMBRAPA Gado de Leite

${ }^{4}$ Aluno de Mestrado em Zootecnia na UFLA.

5 Professor Titular Aposentado do DZO da UFLA e bolsista do CNPq.
} 
tolerados (MARTINEZ, FREITAS E MADALENA, 1979). Mesmo os métodos em que esta compensação de erros não chega a eliminar totalmente as tendências podem ser válidos para ordenar corretamente os animais, em função de seu valor reprodutivo.

Assim, os métodos de controle leiteiro devem ser avaliados conforme os objetivos para os quais são empregados, e não pela distribuição de erros em lactações individuais (McDANIEL, 1969).

Com objetivo de aumentar o número de animais submetidos ao controle leiteiro, reduzir os transtornos operacionais causados na fazenda e o custo do controle leiteiro, propôs-se um método de controle leiteiro simplificado, com base em registros de produção de leite de uma única ordenha, ao invés de registros de duas ordenhas (PORZIO, 1953). POLY e POUTOUS (1967) verificaram que este método é 25 a $30 \%$ mais barato que o controle mensal comum, tendo efeitos mínimos sobre a seleção de vacas e touros, sendo, no entanto, mais preciso que o controle de duas ordenhas.

MARTINEZ et al. (1979), utilizando dados de vacas das raças Holandesa, Jersey e mestiças Holandês-Gir, compararam cinco métodos de controle leiteiro. Foram usados três métodos com produção de duas ordenhas, com freqüência quinzenal, mensal e bimensal, e dois com registro de produção em uma ordenha, alternando mensalmente a da manhã com a da tarde. Os autores concluíram que todos os métodos propiciam resultados similares, ficando a escolha do método na dependência de considerações práticas. O método alternado é mais preciso que o método bimensal de duas ordenhas - 25 a $35 \%$ mais barato (POLY e POUTOUS, 1967). PUTNAM e GILMORE $(1968,1970)$ concluíram que o custo do método alternadoé $20 \%$ menor que o controle mensal em duas ordenhas. DICKHNSON e McDANIEL (1969), estudando lactações de vacas Holandesas, encontraram erro médio em relação à produção real do método alternado e do método mensal tradicional, 2,52 e $1,72 \%$, respectivamente.

Constituiu-se objetivo deste estudo comparar diferentes métodos de controle leiteiro para estimar a produção de leite em até 305 dias de vacas da raça Gir.

\section{Material e Métodos}

Foram usadas 3632 lactações de 2143 vacas da raça Gir, filhas de 336 touros, cujos partos aconteceram no período de 1986 a 1996, distribuídos em 17 rebanhos participantes do programa de Teste de Pro- gênie coordenado pelo Centro Nacional de Pesquisa de Gado de Leite (CNPGL) da Empresa Brasileira de Pesquisa Agropecuária (EMBRAPA) e da Associação Brasileira dos Criadores de Gir Leiteiro (ABCGIL).

Foram consideradas duas estações de parição: os meses de outubro a março constituíram a estação das águas (estação 1) e os de abril a setembro, a estação da seca (estação 2).

Em função da média de produção de leite em até 305 dias, para vacas que pariram com até 46 meses de idade, foram constituídos dois grupos de manejo (baixo = rebanhos com produção de leite menor que $2236 \mathrm{~kg}$ e alto $=$ rebanhos com produção de leite maior ou igual a $2236 \mathrm{~kg}$ ). O número de observações, as médias e os respectivos erros-padrão da produção de leite encontram-se na Tabela 1.

As modificações no arquivo para obtenção de dados consistentes e constituição das classes estudadas, bem como as análises estatísticas, foram feitas por intermédio programa SAS (Statistical Analysis System, 1996).

Foram estudados cinco métodos para estimar a produção de leite na lactação. No método A, o controle na ordenha da manhã foi realizado nos meses ímpares e o controle na ordenha da tarde, nos meses pares. A produção da ordenha da manhã foi multiplicada por $\mathrm{FM}=(\mathrm{PM}+\mathrm{PT}) / \mathrm{PM}$ e a produção da ordenha da tarde, por FT $=(\mathrm{PM}+\mathrm{PT}) / \mathrm{PT}$, em que FM e FT correspondem, respectivamente, a um fator multiplicativo da manhã e da tarde, corrigidos simultaneamente para ano de parto (A), duração da lactação - linear (DL), idade ao parto - linear e quadrática (I) e um fator K, sendo K os efeitos de nível de manejo, classe de ordem de parto (primíparas ou multíparas), estação de parição e classe de intervalo do parto ao controle leiteiro e as possíveis interações duplas, triplas e quadrupla entre estes efeitos.

Nos métodos B e D o controle foi realizado na ordenha da manhã; em B a produção da manhã foi multiplicada pelo fator multiplicativo da manhã (FM) e em D a produção da manhã foi duplicada a cada controle para obtenção da produção de leite no dia do controle $\left(y_{i}\right)$.

Nos métodos $\mathrm{C}$ e E, para obtenção da produção de leite no dia $y_{i}$, o controle foi realizado na ordenha da tarde, sendo que em $\mathrm{C}$ a produção da tarde foi multiplicada pelo fator multiplicativo da tarde (FT) e em E a produção da tarde foi duplicada a cada controle.

A produção total por lactação foi calculada, nos diferentes métodos, pelo produto da média estimada das produções de leite nos controles $y_{i}$, pela duração da lactação $L_{k}$ (A ou B ou C ou D ou 
$\mathrm{E}=\left(L \cdot \sum_{i=1}^{n} y_{i} / \eta\right)($ MARTINEZ et al., 1979).

A produção real (R), obtida pela soma das produções da manhã e da tarde observadas no dia do controle leiteiro, foi utilizada como padrão para se obterem os erros de estimava para cada método. Posteriormente, foi calculada a produção na lactação pelo produto da média estimada das produções nos $\eta$ controles $y_{i}$, pela duração da lactação $\mathrm{L}_{\mathrm{k}}\left(R=L \cdot \sum_{i=n}^{n} y_{i} / \eta\right)$.

\section{Resultados e Discussão}

Média, diferença média em porcentagem da média real, desvios-padrão e coeficientes de variação, das estimativas obtidas pelos diferentes métodos, são apresentados na Tabela 2.

A diferença média para o método $\mathrm{B}$ foi inferior à observada nos demais métodos, indicando que o uso do mesmo para estimar a produção de leite total, neste estudo, não resultaria em estimativa tendenciosa. Este resultado concorda com os relatados na literatura (EVERETT e WANDELL, 1970a e 1970b; SCHAEFFER e RENNIE 1976). Entretanto, o método A apresentou diferença média intermediária entre os métodos B e C. Observa-se que os métodos A, B, C e D superestimaram a produção diária de leite, sendo que o método D o fez em maior valor, visto que apenas duplicou a ordenha da manhã. No método E, em que a ordenha utilizada para estimar a produção de leite no dia do controle leiteiro foi a da tarde, a produção foi subestimada. $\mathrm{O}$ método $\mathrm{B}$, no qual a produção da manhã foi multiplicada pelo fator multiplicativo da manhã (FM), mostrou-se como o melhor para estimar a produção de leite.

A diferença média de $+56 \mathrm{~kg}$ de leite observada quando se comparou o método B com a produção real (R) assemelha-se aos resultados observados por PORZIO (1953), superando os de MARTINEZ et al. (1979), que observaram diferença média de $+23 \mathrm{~kg}$ de leite, quando se utilizaram métodos de controle leiteiro semelhantes aos deste trabalho. Contudo, a diferença média em relação à média real, de $+2,14 \%$, observada no método $\mathrm{B}$, foi inferior à diferença média de $+7,8 \%$, encontrada por NIELSON (1967), citado por MARTINEZ et al. (1979).

A magnitude da diferença média observada nos métodos de controle estudados depende, também, da diferença de produção entre as ordenhas da manhã e da tarde, que, por sua vez, é afetada, principalmente, pelo intervalo de ordenhas (EVERETT e WADELL 1970a, b; SCHAEFFER e RENNIE, 1976). Como neste estudo os intervalos de ordenhas não eram conhecidos, os mesmos podem ter contribuído para incrementar as diferenças médias encontradas nos diferentes métodos.

Os métodos $\mathrm{D}$ e $\mathrm{E}$, nos quais a produção da ordenha da manhã e da tarde, respectivamente, foi duplicada, foram os piores para a estimativa da produção de leite, sugerindo a necessidade de utilização de métodos alternativos que usem de fatores multiplicativos, o que se confirma pela melhor precisão observada nos métodos A, B e C. Estes resultados coincidem com os observados por DICKINSON e McDANIEL (1969).

Na Tabela 2, nota-se que os métodos A e B podem ser utilizados para estimar a produção de leite total, levando a estimativas semelhantes as observadas na produção real $(\mathrm{R})$.

Informações sobre a distribuição das lactações, dentro de determinada porcentagem de erro, estão apresentadas na Tabela 3. O método A e B mostraram-se como os de maior precisão, em que 93 e $89 \%$ das lactações se encontram no intervalo de $\pm 15 \%$ de erro, respectivamente. Observa-se, na Tabela 3, que a precisão dos métodos $\mathrm{A}$ e $\mathrm{B}$ é semelhante, quando se comparou a distribuição das lactações no intervalo de $\pm 25 \%$ de erro. No entanto, quando os métodos $A$, B, C, D e E foram comparados com base na dispersão média dos resíduos de produções de leite estimadas em relação à produção média real (R), Figura 1, a precisão do método A foi intermediária entre os métodos $\mathrm{B}$ e $\mathrm{C}$, sendo superior à dos outros dois métodos (D e E). Estes resultados são atribuídos ao fato de estarem sendo consideradas, para obtenção dos resultados apresentados na Tabela 3, apenas lactações dentro de, no máximo, $\pm 25 \%$ de erro; no entanto, na Figura 1, todas as lactações foram consideradas. Consideradose lactações dentro do intervalo de $\pm 35 \%$ de erros, $100 \%$, das lactações são englobadas pelo método B e as mesmas $99 \%$, englobadas no intervalo de $\pm 25 \%$ de erro, pelo método A, continuam sendo englobadas no intervalo de $\pm 35 \%$ de erros.

Utilizando-se fatores multiplicativos para estimar a produção de leite no dia do controle leiteiro (métodos A, B e C), ao invés de apenas duplicar a produção da ordenha da manhã ou da tarde, como se realizou nos métodos D e E, notou-se redução nos erros de estimativa. Nota-se, ainda, que apenas 69\% das lactações se encontram no intervalo de $\pm 25 \%$ de erro nos métodos D e E. Considerando-se o intervalo de $\pm 15 \%$ de erro, estes métodos englobam apenas $33 \%$ das lactações (Tabela 3). 
Tabela 1 - Média e desvio-padrão da produção de leite em até 305 dias, por nível de manejo Table 1 - Means and standard deviation of the milk yield up to 305 days by management level

Nível de manejo Número de observação Produção de leite

Management level Number of observations Milk production

\begin{tabular}{lccc} 
& & $\begin{array}{c}\text { Média (erro-padrão) } \\
\text { Means (standard error) }\end{array}$ & $\begin{array}{c}\text { Desvio-padrão } \\
\text { Standard deviation }\end{array}$ \\
\hline Alto (High) & 14302 & $2811(7,1)$ & 849 \\
Baixo(Low) & 11135 & $2199(7,4)$ & 780 \\
\hline
\end{tabular}

Tabela 2 - Médias, diferenças médias, desvio-padrão para 3632 lactações estimadas por diferentes métodos

Table 2 - Means, average differences, standard deviation, variation coefficient for 3,632 lactations estimated by different methods

\begin{tabular}{lcccc}
\hline $\begin{array}{l}\text { Método } \\
\text { Method }\end{array}$ & $\begin{array}{c}\text { Média (erro-padrão), } \mathrm{kg} \\
\text { Means (standard error) }\end{array}$ & $\begin{array}{c}\text { Diferença média em } \\
\text { relação à média real (\%) } \\
\text { Average difference relative } \\
\text { to the actual means }\end{array}$ & $\begin{array}{c}\text { Desvio-padrão (kg) } \\
\text { Standard deviation }\end{array}$ & $\mathrm{CV}(\%)$ \\
\hline $\mathrm{R}$ & - & 988 & 37,7 \\
$\mathrm{~A}$ & $2622(6,2)$ & $+4,80$ & 1056 & 38,4 \\
$\mathrm{~B}$ & $2750(6,6)$ & $+2,14$ & 1072 & 40,1 \\
$\mathrm{C}$ & $2678(6,7)$ & $+7,21$ & 1083 & 38,5 \\
$\mathrm{D}$ & $2811(6,8)$ & $+19,1$ & 1264 & 40,5 \\
$\mathrm{E}$ & $3122(7,9)$ & $-19,1$ & 807 & 38,1 \\
\hline
\end{tabular}

Tabela 3 - Porcentagem de lactações estimadas dentro de determinada porcentagem de erro (em valor absoluto), em relação à produção real

Table 3 - Estimated lactation percentages within a given error percentage (in absolute value) in relation to the actual production

\begin{tabular}{lcccccccc}
\hline \multirow{8}{*}{$\begin{array}{l}\text { Método } \\
\text { Method }\end{array}$} & $\mathbf{7}$ & \multicolumn{7}{c}{$\begin{array}{c}\text { Erro(\%) } \\
\text { Error }\end{array}$} \\
\cline { 2 - 10 } A & \pm 2 & \pm 4 & \pm 6 & \pm 8 & \pm 10 & \pm 15 & \pm 20 & \pm 25 \\
B & 19 & 38 & 55 & 71 & 81 & 93 & 98 & 99 \\
C & 13 & 26 & 39 & 51 & 61 & 89 & 97 & 99 \\
D & 11 & 22 & 33 & 43 & 51 & 66 & 75 & 81 \\
E & 13 & 15 & 18 & 20 & 23 & 33 & 49 & 69 \\
\hline
\end{tabular}

Pela análise dos dados da Tabela 4, pode-se inferir que o desvio absoluto em quilogramas de leite segue a mesma tendência da distribuição de erros, para os diferentes métodos. Percebe-se que $87 \%$ das lactações estão dentro do intervalo de $\pm 300 \mathrm{~kg}$ de leite no método A e 75\% no método B. Estes resultados concordam com os observados por MARTINEZ et al. (1979), quando foram usados métodos similares aos deste estudo. Para os métodos D e E, a porcentagem de lactações dentro do intervalo de $\pm 300 \mathrm{~kg}$ de leite foi inferior, apenas $30 \%$ das lactações.

Os coeficientes de correlação de Pearson entre as produções de leite real (R), a produção em até 305 dias (P305), produção total (Ptotal) e as produções estimadas pelos diferentes métodos (A, B, C, D, E) são apresentados na Tabela 5. Constata-se que todas as correlações estimadas entre as produção dos diferentes métodos e a produção em até 305 dias, a produção total e a produção real foram altas. Os valores encontrados estão em concordância com os observados por CASTLE e SEARLE (1961) e MARTINEZ et al. (1979).

Os valores de correlação semelhantes, observados entre os diferentes métodos, não indicam que os 
Rev. bras. zootec.

Tabela 4 - Porcentagem de lactações estimadas dentro de um dado número de kg de leite (em valor absoluto) da produção real

Table 4 - Proportion of estimated lactation within a given milk production in $\mathrm{kg}$ (absolute value) of the actual production

\begin{tabular}{lcccccccc}
\hline \multirow{2}{*}{$\begin{array}{l}\text { Método } \\
\text { Method }\end{array}$} & \pm 50 & \pm 100 & \pm 150 & \pm 200 & \pm 250 & \pm 300 & \pm 350 & \pm 400 \\
\cline { 2 - 8 } & 20 & 40 & 58 & 73 & 82 & 87 & 91 & 93 \\
A & 14 & 28 & 41 & 53 & 65 & 75 & 82 & 87 \\
B & 11 & 23 & 33 & 42 & 50 & 57 & 63 & 68 \\
C & 13 & 16 & 19 & 22 & 26 & 30 & 34 & 40 \\
D & 13 & 16 & 19 & 22 & 26 & 30 & 34 & 40 \\
E & 13
\end{tabular}

Tabela 5 - Correlação de Pearson entre as produções diárias estimadas pelos diferentes métodos e a produção de leite real, a produção em até 305 dias e a produção total

Table 5 - Pearson's correlation between the estimated dairy production by the different methods and the milk actual production, the production at 305 days and the total production

\begin{tabular}{lccccc}
\hline & \multicolumn{5}{c}{ Método (Method) } \\
\cline { 2 - 6 } & $\mathrm{A}$ & $\mathrm{B}$ & $\mathrm{C}$ & $\mathrm{D}$ & $\mathrm{E}$ \\
\hline $\mathrm{R}$ (actual production) & 0,98 & 0,97 & 0,93 & 0,97 & 0,93 \\
P305 & 0,95 & 0,93 & 0,90 & 0,94 & 0,89 \\
Ptotal (Total production) & 0,96 & 0,95 & 0,89 & 0,95 & 0,89 \\
\hline
\end{tabular}

métodos foram equivalentes para a estimativa da produção de leite, e sim que existe dependência linear entre as produções estimadas pelos diferentes métodos e a produção real de leite (LEDIC et al., 1991).

A comparação das correlações entre as produções estimadas e a produção real, quando o controle foi realizado na ordenha da tarde (métodos $\mathrm{C}$ e E), com as observadas em controles realizados na ordenha da manhã (métodos B e D), sugere que, ao se realizar o controle na ordenha da manhã, tem-se melhor estimativa da produção de leite total, obtendo-

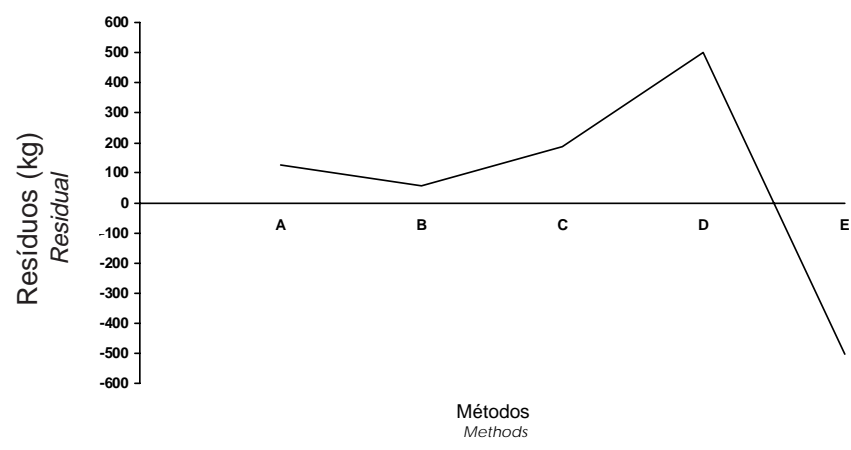

Figura 1 - Dispersão dos resíduos de produções médias de leite estimada por diferentes métodos de controle em relação à produção média real.

Figure 1 - Dispersion of the residues of average productions of milk estimated by different control methods relative to the real average production. se, no entanto, estimativas semelhantes às observadas quando os controles foram alternados entre a ordenha da manhã e tarde (método A).

Nas Figuras 2, 3, 4 e 5, encontra-se a dispersão média dos resíduos de produção diária de leite estimada pelos fatores multiplicativos, em relação à produção diária observada, quando se usou o método A para estimar a produção de leite no dia do controle leiteiro.

Observa-se que os fatores multiplicativos da manhã, usados para estimar a produção de leite, tenderam a superestimar, em pequenos valores, a

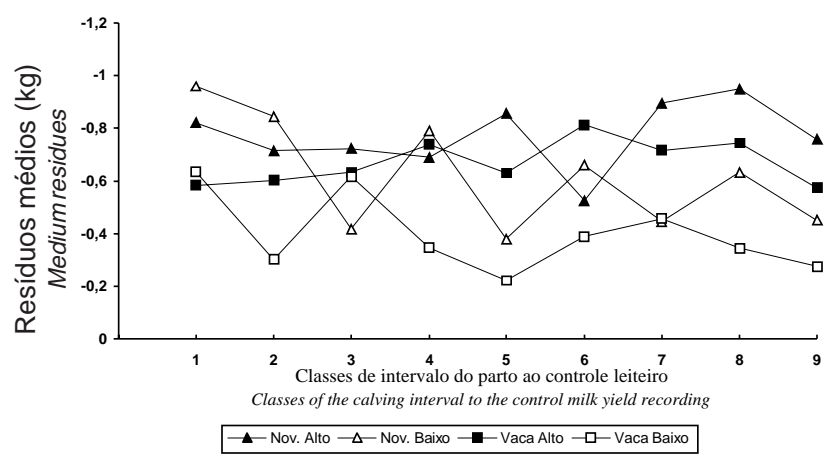

Figura 2 - Dispersão média dos resíduos de produção diária de leite estimada pelos fatores da manhã, quando o controle era realizado em meses ímpares, em relação a produção diária observada. Águas.

Figure 2 - Average dispersion of the residues of daily milk production estimated by the morning factors, when the control was undertaken in odd months, in relation to the observed dairy production. Rainy season. 


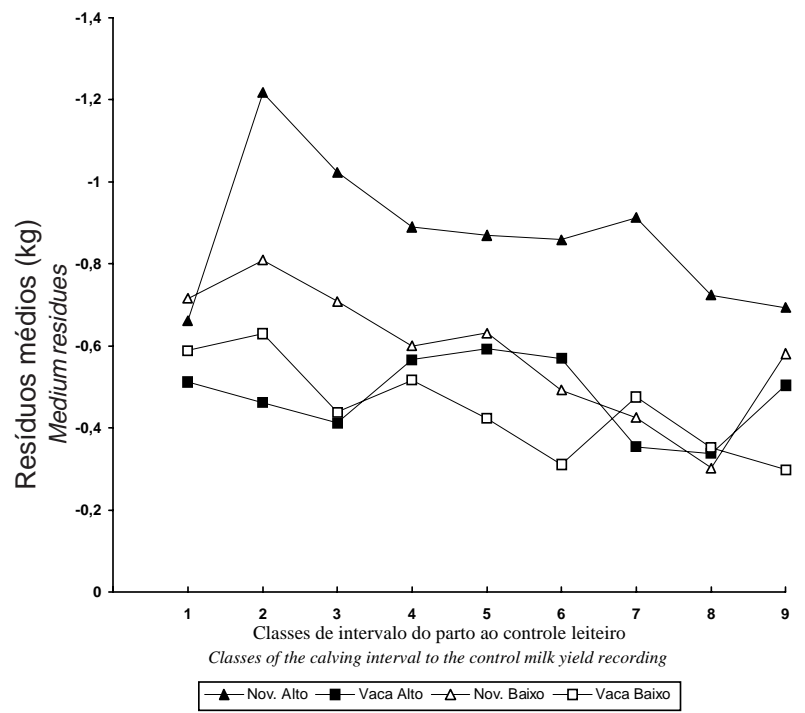

Figura 3 - Dispersão média dos resíduos de produção diária de leite, estimada pelos fatores da manhã, quando o controle foi realizado em meses ímpares, em relação à produção diária observada. Seca.

Figure 3 - Average dispersion of the residues of daily milk production estimated by the morning factors, when the control was undertaken in odd months, in relation to the observed dairy production. Dry season.

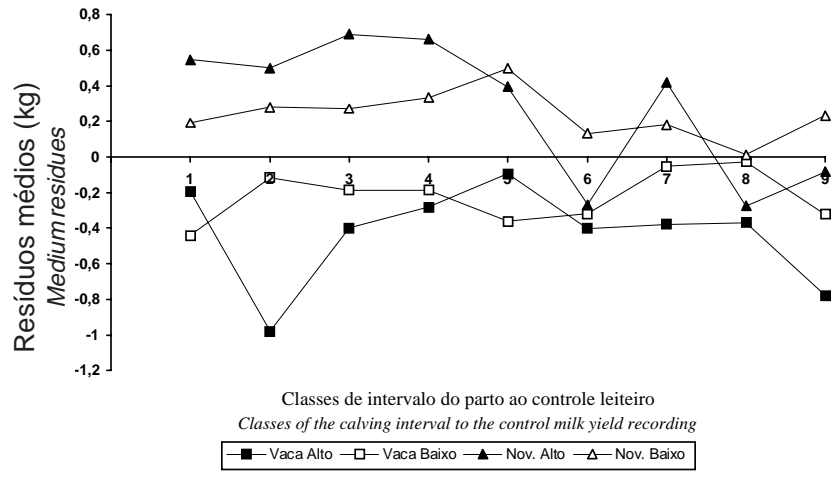

Figura 4 - Dispersão média dos resíduos de produção diária de leite, estimada pelos fatores da tarde, quando o controle foi realizado em meses pares, em relação à produção diária observada. Aguas.

Figure 4 - Average dispersion of the residues of daily milk production estimated by the afternoon factors, when the control was undertaken in even months, in relation to the observed dairy production. Rainy season.

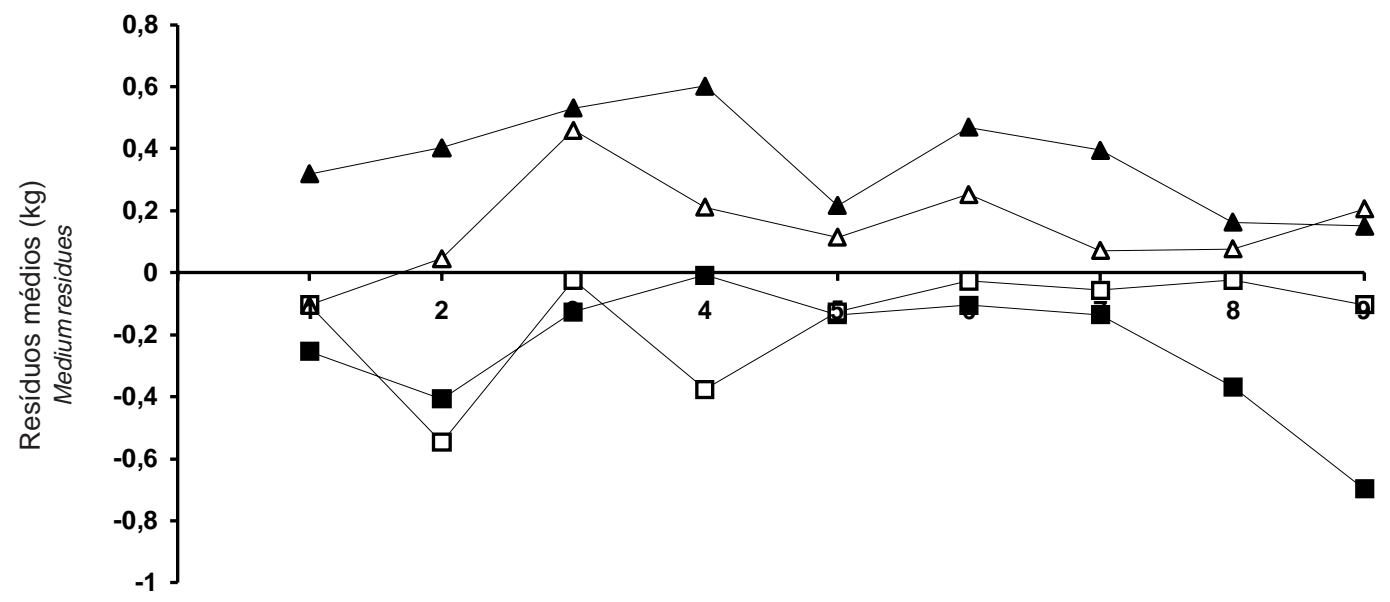

Classes de intervalo do parto ao controle leiteiro

Classes of the calving interval to the control milk yield recording

$\square$ - Vaca Alto $-\mathbf{\square}$ - Vaca Baixo $\longrightarrow$ Nov. Alto $\longrightarrow$ Nov. Baixo

Figura 5 - Dispersão média dos resíduos de produção diária de leite, estimada pelos fatores da tarde, quando o controle foi realizado em meses pares, em relação a produção diária observada. Seca.

Figure 5 - Average dispersion of the residues of daily milk production estimated by the morning factors, when the control was undertaken in even months, in relation to the observed dairy production. Dry season. 
Rev. bras. zootec.

produção de leite no dia do controle leiteiro (Figuras 2 e 3), o que se verifica também para os fatores da tarde em vacas; já os fatores multiplicativos da tarde para novilhas tenderam a subestimar a produção de leite no dia do controle leiteiro (Figuras 4 e 5).

Nas Figuras 2, 3, 4 e 5, observa-se tendência de os fatores multiplicativos da manhã e da tarde, nas duas estações de parição, para vacas mantidas em rebanhos de baixo nível de manejo, estimarem com maior exatidão a produção de leite no dia do controle leiteiro, seguidos dos fatores calculados para vacas mantidas em rebanho de alto nível de manejo. Os fatores calculados para novilhas mostraram-se menos precisos em estimar a produção de leite no dia do controle leiteiro (Figuras 2, 3, 4 e 5). Observa-se, ainda, que, dentro do método de controle $\mathrm{A}$, os fatores multiplicativos que melhor estimaram a produção de leite no dia do controle leiteiro foram os da tarde, para vacas mantidas em rebanhos de baixo nível de manejo, quando o controle foi realizado em meses pares na estação das águas.

\section{Conclusões}

O método A, em que o controle na ordenha da manhã foi realizado nos meses ímpares e o controle na ordenha da tarde, nos meses pares, e a produção da ordenha da manhã foi multiplicada por $\mathrm{FM}=(\mathrm{PM}+\mathrm{PT}) / \mathrm{PM}$ e a produção da ordenha da tarde, por $\mathrm{FT}=(\mathrm{PM}+\mathrm{PT}) / \mathrm{PT}$, apresentou-se como o mais viável para estimar a produção de leite no dia do controle leiteiro, por sua praticidade e precisão. Os métodos de controle em que a produção de uma das ordenhas foi duplicada a cada controle foram os piores métodos de controle leiteiro.

\section{Referências Bibliográficas}

CASTLE, O.M., SEARLE, S.H. 1961. Use of bimonthly records in herd testing. J. Dairy Sci., 44(97):1335-1343.

DICKINSON, F.N., McDANIEL, B.T. 1969. Single-milk yields versus 24-hour yields for estimating lactation milk production by the test interval method. J. Anim. Sci., 53(2):200-207.

EVERETT, R.W., WADELL, L.H. 1970a. Sources of variation affecting the difference between morning and evening dairy milk production. J. Anim. Sci., 53(10):1424-1429.

EVERTT, R.W., WADELL, L.H. 1970b. Sources of variation affecting ratio factors for estimating total daily milk yield from individual milking. J. Dairy Sci., 53(10):1430-1435.

LEDIC, I. L., SOBRINHO, E.B., TUPY, O. 1991. Comparação de alguns métodos de previsão de leite com base em controles semanais, quinzenais, mensais e bimensal. Pesq. Agropec. Bras., 26(8):1127-1137.

MARTINEZ, M.L., FREITAS, A.F., MADALENA, F.E. 1979. Comparação de métodos para estimar a produção de leite com base em controle mensais. Pesq. Agropec. Bras., 14(3):243-250.

McDANIEL, B.T. 1969. Accuracy of sampling procedures for estimating lactation yields; a review. J. Dairy Sci., 52(11):1742-1761.

POLY, J.E., POUTOUS, M. 1967. Le controle laitier mensuel alterne (AT). I. Précision vis-à-vis dún controle mensuel ou bimestriel pour la production de lait en 305 Jours. Ann. Zootech., 16(2):183-190.

PORZIO, G. II 1953. Controlo del latte second un nuovo método. Italia Agricula, Roma, 90:421-427.

PUTNAM, D.N., GILMORE, H.C. 1970. Factors to adjust milk production to a 24 basis when milking intervals are unequal.. J. Dairy Sci., 53:685 (Abst. E9)

PUTNAM, D.N., GILMORE, H.C. 1968. AM PM Evaluation of an alternate AM and PM monthly testing plan and its application for use in the Dairy Herd Improvement Association program. J. Dairy Sci., 51:985, (Abst. E4)

SCHAEFFER, L.R., RENNIE, J.C. AM-PM 1976. Testing for estimating lactation yields. Can. J. Anim. Sci., 56(1):9-15.

STATISTICAL ANALYSIS SYSTEM - SAS. 1996. User's Guide: Statistics, Cary: SAS INSTITUTE. 956p.

Recebido em: 18/05/98

Aceito em: 22/04/99 